\title{
Thioglycosides as inhibitors of hSGLT1 and hSGLT2: Potential therapeutic agents for the control of hyperglycemia in diabetes
}

\author{
Francisco Castaneda ${ }^{1}$, Antje Burse ${ }^{2}$, Wilhelm Boland ${ }^{2}$, Rolf K-H. Kinne ${ }^{1}$ \\ 1. Laboratory for Molecular Pathobiochemistry and Clinical Research, Max Planck Institute of Molecular Physiology, Dort- \\ mund, Germany; \\ 2. Max Planck Institute for Chemical Ecology, Dortmund, Germany
}

Correspondence to: Francisco Castaneda, MD, Laboratory for Molecular Pathobiochemistry and Clinical Research, Max Planck Institute for Molecular Physiology, Otto-Hahn-Str. 11, 44227 Dortmund, Germany; Tel. 49-231-9742-6490, Fax. 49-231-133-2699, E-mail: francisco.castaneda@mpi-dortmund.mpg.de

Received: 2007.04.14; Accepted: 2007.04.30; Published: 2007.05.05

The treatment of diabetes has been mainly focused on maintaining normal blood glucose concentrations. Insulin and hypoglycemic agents have been used as standard therapeutic strategies. However, these are characterized by limited efficacy and adverse side effects, making the development of new therapeutic alternatives mandatory. Inhibition of glucose reabsorption in the kidney, mediated by SGLT1 or SGLT2, represents a promising therapeutic approach. Therefore, the aim of the present study was to evaluate the effect of thioglycosides on human SGLT1 and SGLT2. For this purpose, stably transfected Chinese hamster ovary (CHO) cells expressing human SGLT1 and SGLT2 were used. The inhibitory effect of thioglycosides was assessed in transport studies and membrane potential measurements, using a-methyl-glucoside uptake and fluorescence resonance energy transfer, respectively. We found that some thioglycosides inhibited hSGLT more strongly than phlorizin. Specifically, thioglycoside I (phenyl-1'-thio- $\beta$-D-glucopyranoside) inhibited hSGLT2 stronger than hSGLT1 and to a larger extent than phlorizin. Thioglycoside VII (2-hydroxymethyl-phenyl-1'-thio- $\beta$-D-galacto-pyranoside) had a pronounced inhibitory effect on hSGLT1 but not on hSGLT2. Kinetic studies confirmed the inhibitory effect of these thioglycosides on hSGLT1 or hSGLT2, demonstrating competitive inhibition as the mechanism of action. Therefore, these thioglycosides represent promising therapeutic agents for the control of hyperglycemia in patients with diabetes.

Key words: Thioglycoside, sodium-dependent glucose transport, a-methyl-glucoside uptake, fluorescence resonance energy transfer, diabetes, hyperglycemia

\section{Introduction}

Diabetes mellitus is characterized by reduced insulin secretion from pancreatic $\beta$-cells (type 1 diabetes) [1] or deficient insulin action (type 2 diabetes) [2], both causing an increase in blood glucose concentration. High blood glucose (hyperglycemia) represents the main pathogenic factor for the development of diabetic complications including coronary heart disease, retinopathy, nephropathy, and neuropathy $[3,4]$. In addition, chronic hyperglycemia leads to progressive impairment of insulin secretion and to insulin resistance of peripheral tissues (referred to as glucose toxicity) $[1,2,5,6]$. As a consequence, the treatment of diabetes has been mainly focused on maintaining normal blood glucose levels. For that purpose either insulin or hypoglycemic agents have been used as standard therapeutic agents for the treatment of diabetes [7]. The mechanism of action of the anti-diabetic agents used for the treatment of type 2 diabetes, include increasing insulin release, improving glucose disposal, controlling hepatic glucose release or inhibiting intestinal glucose absorption [8].

Glucose is unable to diffuse across the cell mem- brane and requires transport proteins [9]. The transport of glucose into epithelial cells is mediated by a secondary active cotransport system, the sodium-D-glucose cotransporter (SGLT), driven by a sodium-gradient generated by the $\mathrm{Na}^{+} / \mathrm{K}^{+}$-ATPase. Glucose accumulated in the epithelial cell is further transported into the blood across the membrane by facilitated diffusion through GLUT transporters.

SGLT belongs to the sodium/glucose cotransporter family SLCA5 [10]. Two different SGLT isoforms, SGLT1 and SGLT2, have been identified to mediate renal tubular glucose reabsorption in humans. Both of them are characterized by their different substrate affinity [11]. Although both of them show 59\% homology in their amino acid sequence, they are functionally different. SGLT1 transports glucose as well as galactose, and is expressed both in the kidney and in the intestine, while SGLT2 is found exclusively in the S1 and S2 segments of the renal proximal tubule [11]. As a consequence, glucose filtered in the glomerulus is reabsorbed into the renal proximal tubular epithelial cells by SGLT2, a low-affinity/high-capacity system, in S1 and S2 tubular segments. Much smaller amounts of glucose are recovered by SGLT1, as a 
high-affinity/low-capacity system, in the distal segment of the tubule.

Inhibition of glucose reabsorption in the kidney, mediated by the SGLT cotransport system, represents a promising therapeutic target for the control of hyperglycemia. The rationale to use SGLT as a target resulted from evidence obtained on several in vitro and in vivo animal studies [12-14] that show the efficacy of D-glucose analogues in inhibiting glucose transport [15]. This mechanism leads to increased urinary glucose excretion and consequently reduces blood glucose concentration.

Tsujihara et al. [12] studies using phlorizin, an O-glucoside derivative were published in 1996. Phlorizin is the most studied substance to date [16]. It inhibits the activity of SGLT in the kidney leading to glycosuria [17]. Its clinical application; however, is restricted due to hydrolysis by $\beta$-glucosidases in the intestine [12]. To overcome this problem, phlorizin analogues have been chemical synthesized [13, 14]. The most commonly used is known as T-1095 (3-(benzofuran-5-yl)-2',6'-dihydroxy-4'-methylpropiophenone 2'-O-(6-O-methoxycarbonyl- $\beta$-D-glycopyranoside) [18]. T-1095 is absorbed through the small intestine and converted into its active form, a specific inhibitor of renal SGLT, resulting in inhibition of glucose reabsorption in the renal tubules $[17,19]$. This compound was the first orally administered active agent with anti-hyperglycemic action that was proposed for the treatment of diabetes mellitus, based on studies using diabetic animal models in rats [20-22] and mice [23].

Since SGLT recognizes glucose analogues as a substrate, it is possible that other glucoside derivates could also inhibit the activity of SGLT. The role of glucose analogues on SGLT inhibition has been well demonstrated in vitro $[19,20]$ and in vivo animal models $[17,21-26]$. Among these, thioglycosides are important to consider because they are not hydrolysed by $\beta$-glucosidases in the intestine and can be administered orally [27].

Therefore, the aim of the present study was to evaluate the inhibitory effect of some thioglycosides synthesized in our laboratory on human hSGLT1 and hSGLT2 -as a potential therapeutic alternative for the control of hyperglycemia, particularly for people with diabetes. We chose to analyze the inhibitory effect of thioglucosides on human SGLT1 and 2 expressed in $\mathrm{CHO}$ cells due to their substrate selectivity and the kinetics of SGLT on different species [17, 28].

\section{Materials and Methods}

\section{Cell Culture}

Stably transfected Chinese hamster ovary $(\mathrm{CHO})$ cells, that express human SGLT1 or human SGLT2 established in our laboratory [29], were seeded at a concentration of $1 \times 10^{3}$ cells $/ \mathrm{ml}$ and maintained in culture for 2 days to allow the cells to form a confluent monolayer culture. For transport studies cells were seeded in 96-well microtiter scintiplates (PerkinElmer, Wiesbaden, Germany). For fluorescence resonance energy transfer (FRET) analysis cells were seeded in flat-bottom, poly-D-lysine black-wall, clear bottom, 96-well plates (Becton Dickinson; Heidelberg, Germany).

\section{Thioglycosides}

Thioglycosides are molecules in which a sugar group is bounded through its anomeric carbon to another group via an S-glycoside bond. The alkylglucoside structure of thioglycosides allows the specific recognition of these substances by SGLT [30].

We analyzed seven thioglycosides (Table 1). Thioglycosides are hydrolysis-resistant, synthetic S-analogs of natural O-glucosides involved in the biosynthesis of chrysomelidial and salicin. These substances are synthesized and secreted as part of a defense mechanism used by larvae of beetles (Chrysomelidae). Their synthesis has been previously described [31-33]. For the purpose of the present study the thioglycosides used were selected and grouped based on their differences in the aglycone binding site or in the glucose moiety (glucose-galactose).

\section{Determination of SGLT-mediated \\ $\alpha$-methyl-D-glucopyranoside uptake}

Sodium-dependent transport activity was determined by means of radioactive $\left[{ }^{14} \mathrm{C}\right]$ a-methyl-D-glucopyranoside $\left(\left[{ }^{14} \mathrm{C}\right] \mathrm{AMG}\right.$, spec. radioactivity $300 \mathrm{mCi} / \mathrm{mmol}$ ) purchased from NEN (Bad Homburg, Germany), using the 96-well semi-automated method previously described in our laboratory [29]. AMG, a non-metabolizable glucose analogue that is selectively transported through SGLT but not through GLUT transporters, was used. Krebs-Ringer-Henseleit (KRH) solution containing 120 $\mathrm{mM} \mathrm{NaCl}, 4.7 \mathrm{mM} \mathrm{KCl}, 1.2 \mathrm{mM} \mathrm{MgCl} 2,2.2 \mathrm{mM} \mathrm{CaCl}_{2}$, $10 \mathrm{mM}$ HEPES (pH 7.4 with Tris) was used to asses active glucose transport in the presence of sodium. For sodium free conditions, KRH solution containing 120 $\mathrm{mM}$ N-methyl-glucamine (NMG) instead of $\mathrm{NaCl}$ $\left(\mathrm{Na}^{+}\right)$was used to assess the sodium-independent D-glucose transport (SGLT). The difference between the two experimental setups represents the sodium-dependent transport by hSGLT1 or hSGLT2. All chemicals were purchased from Sigma (Deisenhofen, Germany).

Briefly, cells were rinsed three times with $200 \mu \mathrm{l}$ $\mathrm{KRH}-\mathrm{Na}^{+}$or KRH-NMG. Then, $100 \mu \mathrm{l}$ pro well of transport buffer containing $\mathrm{KRH}-\mathrm{Na}^{+}$or $\mathrm{KRH}-\mathrm{NMG}$ plus $\left[{ }^{14} \mathrm{C}\right] \mathrm{AMG}(0.1 \mu \mathrm{Ci} / \mu \mathrm{l})$ were added and the cells incubated for $1 \mathrm{~h}$. At the end of the uptake period, $\left[{ }^{14} \mathrm{C}\right] \mathrm{AMG}$-uptake was stopped by adding $100 \mu \mathrm{l}$ of ice-cold stop buffer $\left(\mathrm{KRH}-\mathrm{Na}^{+}\right.$, containing $0.5 \mathrm{mM}$ phlorizin). Then, the cells were solubilized by adding $100 \mu \mathrm{l}$ of ATPlite substrate solution (PerkinElmer, Boston, USA), and luminescence for ATP detection was assessed using a MicroBeta Trilux (PerkinElmer). A standard curve was used to determine the amount of ATP in mg of protein measured from the number of cells per well. After $24 \mathrm{~h}$, the microtiter plate was taken for scintillation counting of radioactive $\left[{ }^{14} \mathrm{C}\right] \mathrm{AMG}$ using a MicroBeta Trilux (PerkinElmer). 
Subsequently, the mean counts per minute (cpm) were calculated and converted to picomoles (pmol). Uptake was expressed as $\mathrm{pmol} / \mathrm{mg} / \mathrm{h}$. Sodium-dependent $\left[{ }^{14} \mathrm{C}\right] \mathrm{AMG}$ uptake was calculated by subtracting uptake under sodium-free conditions from the uptake obtained in the presence of sodium. Results are ex- pressed as percent of inhibition from AMG uptake in CHO cells expressing hSGLT1 or hSGLT2 but not exposed to thioglycosides. $\mathrm{IC}_{50}$ values were calculated using the Kinetic Enzyme Module (SigmaPlot 8.02, Systat Software, Erkrath, Germany).

Table 1 Thioglycosides used to evaluate their inhibitory effect on hSGLT1 and hSGLT2<smiles>Oc1ccccc1</smiles>

I<smiles>Cc1ccccc1S(C)(Cl)Cl</smiles>

II<smiles>OCc1ccccc1S(Cl)(Cl)Cl</smiles><smiles>O=S(=O)(Cl)c1ccccc1CO</smiles>

VII<smiles>Cc1cccc(S(Cl)(Cl)Cl)c1</smiles>

III<smiles>O=S(=O)(Cl)c1cccc(CO)c1</smiles><smiles>CC1=CC=C(S(C)(=O)=O)CC1</smiles>

IV

\begin{tabular}{|c|c|}
\hline & Thioglycoside \\
\hline 1 & Phenyl-1'-thio- $\beta$-D-glucopyranoside \\
\hline II & o-Tolyl-1'-thio- $\beta$-D-glucopyranoside \\
\hline III & $m$-Tolyl-1'-thio- $\beta$-D-glucopyranoside \\
\hline IV & $p$-Tolyl-1'-thio- $\beta$-D-glucopyranoside \\
\hline V & 2-Hydroxymethyl-phenyl-1'-thio- $\beta$-D-glucopyranoside \\
\hline $\mathrm{VI}$ & 3-Hydroxymethyl-phenyl-1'-thio-ß-D-glucopyranoside \\
\hline VII & 2-Hydroxymethyl-phenyl-1'-thio- $\beta$-D-galactopyranoside \\
\hline
\end{tabular}

\section{Measurement of SGLT-mediated thioglycoside translocation}

SGLT-mediated translocation of thioglycosides was determined by assessing the membrane depolarization using fluorescence resonance energy transfer (FRET). Cells were incubated for $48 \mathrm{~h}$ at $37^{\circ} \mathrm{C}$ in a $5 \%$ $\mathrm{CO}_{2}$ in growth medium. Subsequently, cells were washed with $0.2 \mathrm{ml}$ Dulbecco's phosphate-buffered saline (PBS; Invitrogen, Karlsruhe, Germany) and then incubated with $0.1 \mathrm{ml}$ of a solution containing $5 \mu \mathrm{M}$ CC2-DMPE and $0.02 \%$ pluronic acid in PBS. After in- cubation in the dark for $30 \mathrm{~min}$ at $25^{\circ} \mathrm{C}$, cells were washed twice with $0.2 \mathrm{ml}$ PBS. After that, cells were incubated in the dark at $25^{\circ} \mathrm{C}$ for $30 \mathrm{~min}$ with $0.1 \mathrm{ml}$ of a solution containing $1 \mu \mathrm{M} \operatorname{DiSBAC}_{2}(3)$. At the end of the incubation period the wells were excited by 390 $\mathrm{nm}$. Fluorescence emission was recorded at 460 and $580 \mathrm{~nm}$. After a $20 \mathrm{sec}$ baseline reading, $0.1 \mathrm{ml}$ of PBS containing $10 \mu \mathrm{M}$ of the compound investigated was added, and the fluorescence signal was recorder for 40 sec. The change in fluorescence was calculated as the ratio of $\mathrm{F} / \mathrm{F}_{0}$ equal to $=\left[\left(\mathrm{A}_{460} / \mathrm{A}_{580}\right) /\left(\mathrm{I}_{460} / \mathrm{I}_{580}\right)\right]$, where $\mathrm{A}$ and I represent the readings after or before addition each thioglucoside, respectively. For I, the readings 
from 2-5 sec were averaged; and for A, readings from $3 \mathrm{sec}$ after the signal had reached a plateau level (usually within 2-5 sec) were also averaged. FRET values were expressed as relative fluorescence units (RFU).

\section{Statistical analysis}

Data are expressed as mean values \pm standard deviation (SD). Results of $\left.{ }^{[14} \mathrm{C}\right] \mathrm{AMG}$ uptake in the stably transfected $\mathrm{CHO}$ cells treated with each thioglycoside were compared with $\left[{ }^{14} \mathrm{C}\right] \mathrm{AMG}$ uptake in $\mathrm{CHO}$ cells not exposed to thioglycoside (control cells) using independent t-test analysis, and expressed as percent inhibition from uptake in control cells. The change in fluorescence resonance energy transfer (FRET) signal was normalized to the values obtained from non-transfected $\mathrm{CHO}$ cells, and compared to control cells using independent t-test analysis. Statistical significance was assumed at $p$ level $<0.05$ level. SigmaPlot software version 8.02 (Systat Software, Erkrath, Germany) was used for statistical analysis.

\section{Results}

\section{Inhibition of SGLT transport activity}

The thioglycosides investigated in this study are shown in Table 1. Figure 1 shows the inhibitory effect of each thioglycoside $(10 \mu \mathrm{M})$ and phlorizin $(10 \mu \mathrm{M})$ on sodium-dependent AMG-uptake in hSGLT1 and hSGLT2, as compared to control CHO cells. The AMG concentration was $3 \mu \mathrm{M}$. As expected all thioglycosides inhibited sodium-dependent AMG-uptake. In most cases the inhibitory effect was similar both with regard to the two transporters (hSGLT1 and hSGLT2) and to the inhibition exerted by the same concentration of phlorizin, exceptions are thioglycosides I and VII. Thioglycoside I inhibited hSGLT2 stronger than hSGLT1 and to a larger extent than phlorizin; while thioglycoside VII had a more pronounced inhibitory effect on hSGLT1 than on hSGLT2 $(p<0.01)$.

The inhibitory effect of thioglycoside I was stronger for hSGL2 than for hSGLT1, with values of $66.7 \pm 3.2 \%$ and $23.2 \pm 2.8 \%$, respectively. In contrast, thioglycoside VII had a higher inhibitory effect on hSGLT1 than hSGLT2 with values of $57.9 \pm 2.3 \%$ and $26.7 \pm 1.9 \%$, respectively. These values were higher compared to those obtained with phlorizin $(10 \mu \mathrm{M})$, which were equivalent to $34.8 \pm 1.6 \%$ inhibition for hSGLT1 and $33.4 \pm 1.8 \%$ inhibition for hSGLT2. These findings suggest that thioglycosides I and VII have a strong inhibitory effect on hSGLT2 and hSGLT1, respectively.

To analyze further the inhibitory effect on sodium-dependent AMG-uptake of each thioglycoside, $\mathrm{IC}_{50}$ values were determined. As shown in Table 2, the $\mathrm{IC}_{50}$ values of all seven thioglycosides ranged from 9 $\mu \mathrm{M}$ to $37 \mu \mathrm{M}$ for hSGLT1 and from $10 \mu \mathrm{M}$ to $88 \mu \mathrm{M}$ for hSGLT2. The values obtained by the thioglycosides were similar to those obtained with phlorizin, which were equivalent to $42 \mu \mathrm{M}$ and $28 \mu \mathrm{M}$ for hSGLT1 and hSGLT2, respectively. The inhibition of the sodium-dependent AMG-uptake for all thioglycosides was similar to that obtained using phlorizin, suggest- ing a similar inhibitory effect for all these substances. Figure 2 shows the $\mathrm{IC}_{50}$ curves for thioglycosides I and VII. Thioglycoside I showed IC $_{50}$ values of $30 \mu \mathrm{M}$ for hSGLT1 and $10 \mu \mathrm{M}$ for hSGLT2, while thioglycoside VII showed $\mathrm{IC}_{50}$ values of $15 \mu \mathrm{M}$ for hSGLT1 and 88 $\mu \mathrm{M}$ for hSGLT2. These data confirm the strong inhibitory effects of thioglycoside I and VII on hSGLT2 and hSGLT1, respectively. This finding suggests that these two thioglycosides may be promising anti-diabetic agents, based on their strong inhibitory effects on hSGLT.

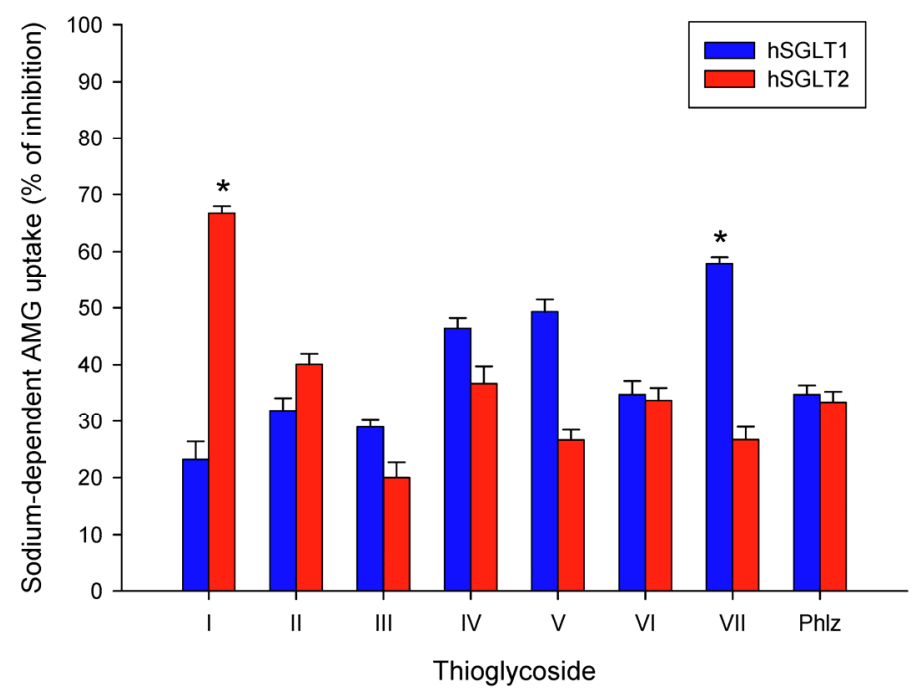

Figure 1 Effect on sodium-dependent $\left[{ }^{14} \mathrm{C}\right] \mathrm{AMG}$-uptake obtained in hSGLT1 or hSGLT2 treated with thioglycosides (10 $\mu \mathrm{M}$ each) or phlorizin $(10 \mu \mathrm{M})$. Results are expressed as percent of inhibition based on uptake in CHO cells expressing hSGLT1 or hSGLT2 not exposed to thioglycosides (control cells). Blue and red bars represent hSGLT1 and hSGLT2, respectively. Results are the mean of six different experiments. Error bars represents standard deviations. ${ }^{*} p<0.01$ shows significantly higher inhibition of sodium-dependent AMG uptake in treated cells as compared to control cells. Control uptake in CHO cells expressing hSGLT1 was $735 \mathrm{pmol} / \mathrm{mg} / \mathrm{h} \pm 22 \mathrm{pmol} / \mathrm{mg} / \mathrm{h}$ and in CHO cells expressing hSGLT2 was $342 \mathrm{pmol} / \mathrm{mg} / \mathrm{h} \pm 15$ $\mathrm{pmol} / \mathrm{mg} / \mathrm{h}$.

Table 2 Inhibitory concentration $\left(\mathrm{IC}_{50}\right)$ of thioglycosides on hSGL1 and hSGLT2, values are expressed as $\mu \mathrm{M}$.

\begin{tabular}{|c|c|c|}
\hline & hSGLT1 & hSGLT2 \\
\hline Thioglycoside & 30 & 10 \\
\hline I & 37 & 42 \\
\hline II & 11 & 40 \\
\hline III & 35 & 52 \\
\hline IV & 9 & 32 \\
\hline V & 12 & 52 \\
\hline VI & 15 & 88 \\
\hline VII & 42 & 28 \\
\hline Phlorizin & & \\
\hline
\end{tabular}



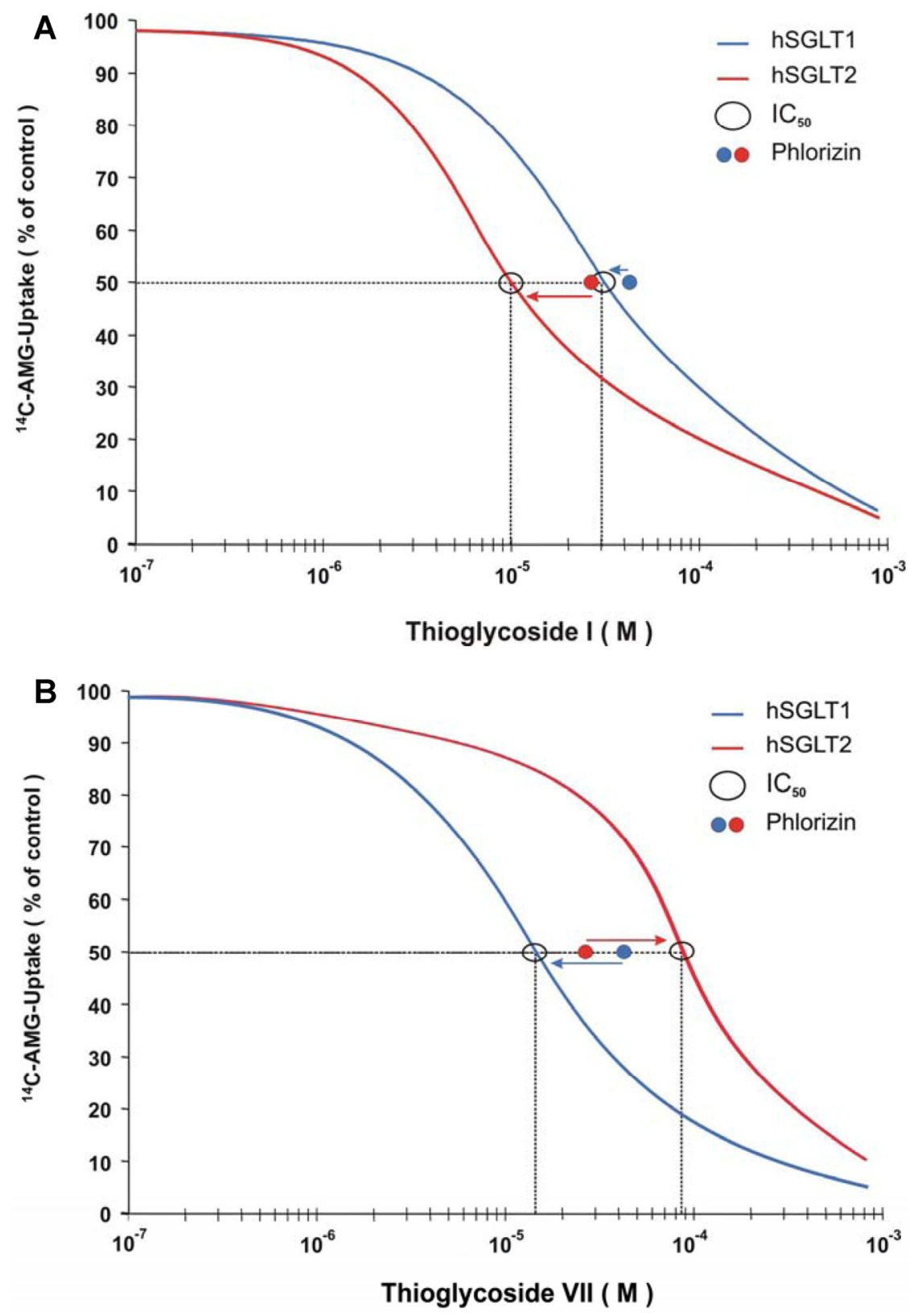

Figure 2 Effect of thioglycoside I and thioglycoside VII on sodium-dependent AMG uptake on CHO cells expressing hSGLT1 (A) and $\mathrm{CHO}$ cells expressing hSGLT2 (B) was determined by $\mathrm{IC}_{50}$ assessment. Different concentrations of thioglycoside I and VII in $\log$ scale were plotted against $\left[{ }^{14} \mathrm{C}\right] \mathrm{AMG}$ uptake as percentage of CHO control cells. The curves for hSGLT 1 and 2 on each cell type were constructed from results from eight different concentrations ranging from $10^{-7}$ to $5 \times 10^{-4}$. The $\mathrm{IC}_{50}$ values of phlorizin are shown as a known reference inhibitory effect.

\section{SGLT Translocation Activity}

In order to investigate whether the thioglycosides were translocated into the cells by the SGLT cotransport system, their effect on membrane potential was measured.-Changes in membrane potential induced by each thioglycoside $(10 \mu \mathrm{M})$ were determined by fluorescence resonance energy transfer (FRET). FRET values were normalized using the change in fluorescence signal obtained from non-transfected $\mathrm{CHO}$ cells. A fluorescence response ratio lower than 1 indicates that these compounds were not significantly transported, while a ratio greater than 1 demonstrates transport across the plasma membrane mediated by SGLT. To validate this assay, studies with D-glucose as a substrate of SGLT were performed and the correlation of sodium-dependent D-glucose uptake to sugar-induced cell membrane depolarization, as measured by FRET, was calculated. As shown in Figure 3, a statistically significant linear relation between the changes in membrane potential and the transport activity of the cells was observed with a correlation coefficient of 0.92 , validating the experimental approach chosen. 


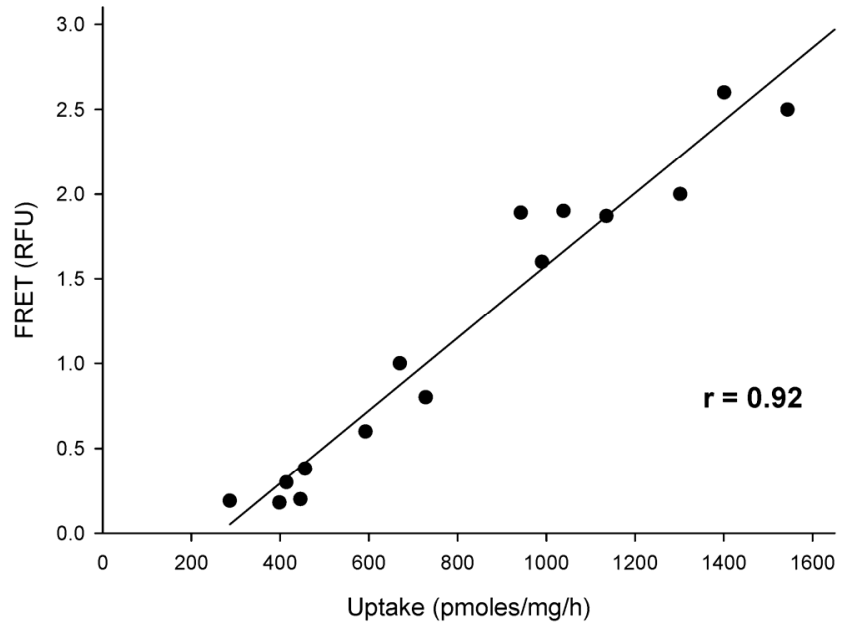

Figure 3 Correlation of sodium-dependent AMG-uptake to sugar-induced cell membrane depolarization is shown. The correlation coefficient of 0.92 demonstrates a strong linear relationship between the two variables $(p<0.001)$.

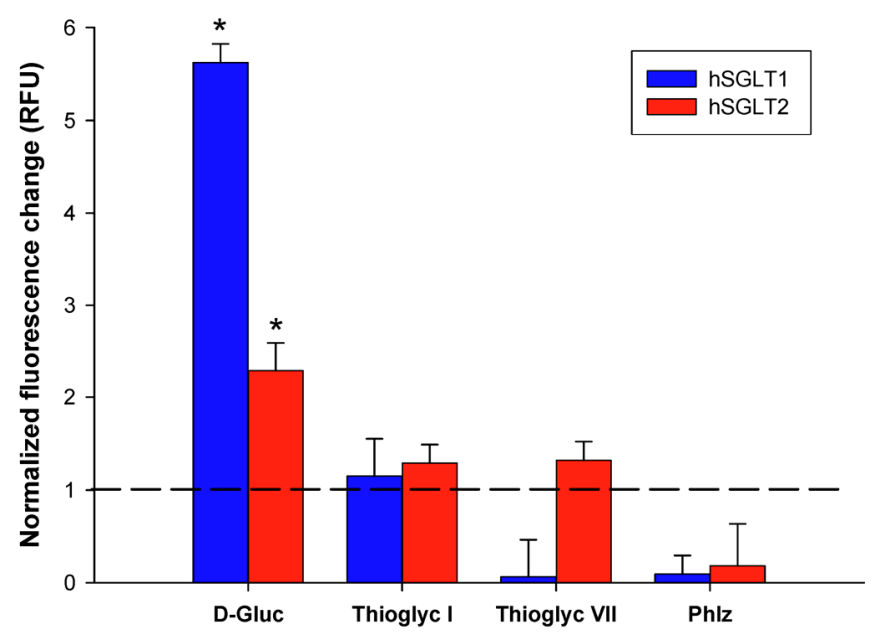

Figure 4 Changes in cell membrane potential induced by D-glucose, thioglycosides I and VII (10 <mM each), and phlorizin were assessed by fluorescence resonance energy transfer (FRET) an expressed as relative fluorescence units (RFU). Blue and red bars represent hSGLT1 and hSGLT2, respectively. The change in FRET signal was normalized to the values obtained from non-transfected $\mathrm{CHO}$ cells (controls). Results are the mean of six different experiments. Error bars represents standard deviations. ${ }^{*} p<0.01$ shows significantly higher induction of cell membrane depolarization in treated cells as compared to control cells (not exposed to thioglycosides).

Figure 4 shows the results in membrane potential induced by D-glucose, thioglycosides I and VII and phlorizin. As expected, the maximal effect was observed with D-glucose with FRET values of 5.62 and 2.29 for hSGLT1 and hSGLT2, respectively. Thioglycoside I showed a small but significant change in cell membrane depolarization with a fluorescence signal ratio of 1.15 for hSGLT1 and 1.29 for hSGLT2 $(p<0.01)$. Thioglycoside VII also showed a significant induction of cell membrane depolarization with a fluorescence ratio of 1.32 for hSGLT2 $(p<0.01)$ while no change was observed for hSGLT1. In contrast, phlorizin was not transported by either hSGLT, as shown by FRET values of 0.09 and 0.18 for hSGLT1 and hSGLT2, respectively. These data clearly show the electrogenic uptake of thioglycoside I and VII across the plasma membrane.

\section{Discussion}

The sodium-dependent glucose transport (SGLT) system represents an excellent target for the development of innovative substances to effectively manage hyperglycemia, thus preventing the adverse complications of glucose toxicity observed in diabetes. The results of the present study suggest that some thioglycosides have a therapeutic potential for the control of blood glucose levels. This promising effect resulted from the strong inhibitory effect of thioglycosides I and VII we observed on sodium-dependent AMG uptake in CHO cells expressing hSGLT2 and hSGLT1, respectively.

Current strategies to treat diabetes are mainly focused in different interventions directed to improve glucose disposal (using insulin sensitizers like metformin), to reduce insulin resistance (using glitazones like rosiglitazone and pioglitazone) and/or to control hepatic glucose release (using biguanides) [7]. In addition, manipulation of insulin through exogenous insulin administration or increase of endogenous insulin production, using sulfonylureas and meglitinides, are also used to treat diabetes [8]. Another diabetes therapeutic approach is based on reducing intestinal glucose absorption using a-glucosidase inhibitors such as acarbose, miglitol and voglibose [34]. a-glucosidase are key enzymes involved in the digestion of carbohydrates.

Inhibition of glucose transport in the kidney through O-glycosides represents a different mechanism of action from other hypoglycemic agents. However, until now it has not been applied to clinical practice because absorption of these anti-hyperglycemic substances (namely phlorizin) is low when administered orally. Studies using alkyl thioglycosides have demonstrated that these substances have a higher affinity for SGLT than O-glucosides [35]. Furthermore, thioglycosides are not metabolized in the intestine [27]. Therefore, a chemical reaction to convert the substance in its active form, after intestinal absorption like in the case of O-glucosides, is not necessary. In addition, alkyl thioglucosides have demonstrated to posses a high renal selectivity [36]. These characteristics make thioglycosides good candidate substances for the control of hyperglycemia. However, their inhibitory effect on a model system for sodium-dependent glucose transport has not been determined, thus the aim of the present study.

We tested whether some alkyl thioglucosides would have the ability to reduce glucose transport through hSGLT1 or hSGLT2 as shown by AMG uptake studies. Our results demonstrate a strong inhibi- 
tory effect of thioglycoside I (phenyl-1'-thio- $\beta$-D-glucopyranoside) on hSGLT2 and thioglycoside VII (2-hydroxymethyl-phenyl-1'-thio- $\beta$-D-galactopyranosi de) on hSGLT1. Studies in animals $[23,37]$ and in humans [38] have demonstrated a higher renal glucose reabsorption in diabetes compared to non diabetic conditions. This has been attributed to an increased expression of GLUT2 transport protein or an increased glomerular filtration rate [39], which may contribute to enhanced glucose transport across the contra luminal membrane and exacerbated hyperglycemia. The reduction in glucose uptake at the luminal site by inhibition of SGLT would effectively decrease glucose reabsorption in the proximal tubule and contribute to control the increased blood glucose levels observed in diabetes. This assumption supports the notion of using SGLT inhibitors as a means to control circulating levels of glucose. The selective inhibition of hSGLT2 by thioglycoside I, which is responsible for most of the reabsorption of glucose in the kidney, represents a promising alternative for the control of hyperglycemia.

It has been shown previously that SGLT1 has a lower affinity to phlorizin than SGLT2 [40]. Based on the $\mathrm{IC}_{50}$ values obtained with phlorizin in the $\mathrm{CHO}$ cells expressing hSGLT1 or hSGLT2, it can be concluded that the method we use is suitable to differentiate between hSGLT1 and hSGLT2. As a consequence, the data obtained by thioglucoside I (phenyl-1'-thio- $\beta$-D-glucopyranoside) and VII (2-hydroxymethyl-phenyl-1'-thio- $\beta$-D-galactopyranosi de) on inhibition of AMG uptake represent important new evidence to be studied further as an alternative for the control of blood glucose levels in diabetic models.

The mechanism of action by which thioglycosides exert this inhibitory effect may be different from that of other oral anti-diabetic agents. Studies using rat enterocytes, where the process of glucose transport is very similar to that in the renal proximal tubule, have demonstrated that glucagon increases SGLT-mediated glucose uptake [41]. Glucagon also promotes GLUT-mediated glucose transport across the proximal tubule [42]. This information suggests that renal glucose reabsorption may be regulated by glucagon and that the direct inhibition of SGLT may represent a viable mechanism for the control of hyperglycemia that is independent from the well known hormonal regulation of blood glucose levels. The change in membrane potential induced by thioglycosides I and VII we found, suggests a competitive mechanism in which each thioglycoside binds to SGLT but are not transported.

Our results also suggest the following structure-activity relationship. We found that thioglucoside I (phenyl-1'-thio- $\beta$-D-glucopyranoside) significantly inhibited hSGLT2-mediated AMG-uptake but to lesser extent hSGLT1, suggesting that differences in the aglycon binding site play an important role in the inhibitory effect of this substance. In contrast, differences in the sugar binding site resulted in an preferen- tial inhibitory effect of thioglycoside VII on hSGLT1 which accepts D-galactose more avidly than D-glucose. This suggests that the glucose moiety may enable different thioglycosides to selectively inhibit active glucose transported mediated by either hSGLT1 or hSGLT2.

Phenyl-O-glucosides have been shown to behave as transported substrates, non-transported inhibitors or non-interacting compounds, depending on the nature and position of the chemical group in the phenyl ring [43]. According to our studies the translocation of thioglycosides seems to be insignificantly, but has to be tested further when radioactively labelled derivatives become available.

The 96-well method used in the present study [29] has the advantage to be able to analyze simultaneously several substances and more importantly, the small concentrations of a given substance required make it possible to test a wide range of substances. However, it must be noted that for hydrophobic compounds due to absorption to the cells and their support, such as thioglycosides, the exact amount of the substance present in a solution cannot be quantified. Thus the $\mathrm{IC}_{50}$ values probably are shifted to higher apparent concentrations.

One of the advantages of using glucose analogues (such as T-1095) to control hyperglycemia has been reported in studies with diabetic rats, in which a significant reduction in diabetic neuropathy has been shown [25]. This finding supports the use of other glucose analogues, such as the thioglycosides we studied, for the treatment of hyperglycemia. In contrast, a possible side effect of thioglycoside treatment is glycosuria. This side effect may resemble the renal glycosuria observed in non-functioning mutations of the SGLT2 gene, leading to a complete absence of renal tubular glucose reabsorption accompanied by increased urinary glucose excretion [44]. However, it has been shown that long term renal glycosuria is not a causative factor for the development of renal damage [44]. Additional studies are needed to confirm the benefits and adverse effects of thioglycoside treatment in humans.

In conclusion, thioglycosides represent promising therapeutic agents for the control of hyperglycemia. In addition, thioglycosides can be used orally, based on its transport in the intestine across the plasma membrane through SGLT1. Thioglycosides have a high renal specificity that is associated with a strong competitive inhibitory effect of sodium-D-glucose cotransporter system mediated by SGLT2. The clinical application of these thioglycosides, however, needs to be further analyzed. Nonetheless, our findings provide the foundation for future studies with the objective to determine the clinical applications of thioglucosides in human diseases like diabetes.

\section{Acknowledgments}

We thank C. Pfaff and P. Glitz for their valuable support in cell culture. 


\section{Conflict of interest}

The authors have declared that no conflict of interest exists.

\section{References}

1. Del Prato S, Matsuda M, Simonson DC, et al. Studies on the mass action effect of glucose in NIDDM and IDDM: evidence for glucose resistance. Diabetologia. 1997; 40: 687-697.

2. Bonadonna RC. Alterations of glucose metabolism in type 2 diabetes mellitus. An overview. Rev Endocr Metab Disord. 2004; 5: 89-97.

3. Klein R. Hyperglycemia and microvascular and macrovascular disease in diabetes. Diabetes Care. 1995; 18: 258-268.

4. Haffner SJ, and Cassells H. Hyperglycemia as a cardiovascular risk factor. The American Journal of Medicine. 2003; 115: 6S-11S.

5. Porte D Jr, and Schwartz MW. Diabetes complications: why is glucose potentially toxic? Science. 1996; 272: 699-700.

6. Robertson RP, Harmon J, Tran PO, et al. Glucose toxicity in beta-cells: type 2 diabetes, good radicals gone bad, and the glutathione connection. Diabetes. 2003; 52: 581-587.

7. Bell DS. Type 2 diabetes mellitus: what is the optimal treatment regimen? Am J Med. 2004; 116: 23S-29S.

8. Wagman AS, and Nuss JM. Current therapies and emerging targets for the treatment of diabetes. Current Pharmaceutical Design. 2001; 7: 417-450.

9. Wood IS, and Trayhurn P. Glucose transporters (GLUT and SGLT): expanded families of sugar transport proteins. British Journal of Nutrition. 2003; 89: 3-9.

10. Wright EM, and Turk E. The sodium/glucose cotransport family SLC5. Pflugers Arch. 2004; 447: 510-518.

11. Wright EM. Renal $\mathrm{Na}(+)$-glucose cotransporters. American Journal of Physiology: Renal Physiology. 2001; 280: F10-F18.

12. Tsujihara $\mathrm{K}$, Hongu $\mathrm{M}$, Saito $\mathrm{K}$, et al. $\mathrm{Na}(+)$-glucose cotransporter inhibitors as antidiabetics. I. Synthesis and pharmacological properties of 4'-dehydroxyphlorizin derivatives based on a new concept. Chem Pharm Bull (Tokyo). 1996; 44: 1174-1180.

13. Hongu $\mathrm{M}$, Tanaka $\mathrm{T}$, Funami $\mathrm{N}$, et al. $\mathrm{Na}(+)$-glucose cotransporter inhibitors as antidiabetic agents. II. Synthesis and structure-activity relationships of 4'-dehydroxyphlorizin derivatives. Chemical \& Pharmaceutical Bulletin (Tokyo). 1998; 46: 22-33.

14. Hongu M, Funami N, Takahashi $\mathrm{Y}$, et al. $\mathrm{Na}(+)$-glucose cotransporter inhibitors as antidiabetic agents. III. Synthesis and pharmacological properties of 4'-dehydroxyphlorizin derivatives modified at the $\mathrm{OH}$ groups of the glucose moiety. Chemical \& Pharmaceutical Bulletin (Tokyo). 1998; 46: 1545-1555.

15. Asano T, Ogihara T, Katagiri $\mathrm{H}$, et al. Glucose transporter and $\mathrm{Na}+$ /glucose cotransporter as molecular targets of anti-diabetic drugs. Curr Med Chem. 2004; 11: 2717-2724.

16. Ehrenkranz JR, Lewis NG, Kahn CR, et al. Phlorizin: a review. Diabetes Metab Res Rev. 2005; 21: 31-38.

17. Nunoi K, Yasuda K, Adachi T, et al. Beneficial effect of T-1095, a selective inhibitor of renal $\mathrm{Na}+$-glucose cotransporters, on metabolic index and insulin secretion in spontaneously diabetic GK rats. Clinical and Experimental Pharmacology and Physiology. 2002; 29: 386-390.

18. Tsujihara $\mathrm{K}$, Hongu $\mathrm{M}$, Saito $\mathrm{K}$, et al. $\mathrm{Na}(+)$-glucose cotransporter (SGLT) inhibitors as antidiabetic agents. 4. Synthesis and pharmacological properties of 4'-dehydroxyphlorizin derivatives substituted on the B ring. Journal of Medicinal Chemistry. 1999; 42: 5311-5324.

19. Ohsumi K, Matsueda $H$, Hatanaka $T$, et al. Pyrazole-O-glucosides as novel $\mathrm{Na}(+)$-glucose cotransporter (SGLT) inhibitors. Bioorganic\& Medicinal Chemistry Letters. 2003; 13: 2269-2272.

20. Oku A, Ueta K, Arakawa K, et al. T-1095, an inhibitor of renal $\mathrm{Na}+$-glucose cotransporters, may provide a novel approach to treating diabetes. Diabetes. 1999; 48: 1794-1800.
21. Oku A, Ueta K, Nawano M, et al. Antidiabetic effect of T-1095, an inhibitor of $\mathrm{Na}(+)$-glucose cotransporter, in neonatally streptozotocin-treated rats. European Journal of Pharmacology. 2000; 391: 183-192.

22. Oku A, Ueta K, Arakawa K, et al. Antihyperglycemic effect of T-1095 via inhibition of renal Na+-glucose cotransporters in streptozotocin-induced diabetic rats. Biological and Pharmaceutical Bulletin. 2000; 23: 1434-1437.

23. Arakawa $\mathrm{K}$, Ishihara $\mathrm{T}$, Oku A, et al. Improved diabetic syndrome in C57BL/KsJ-db/db mice by oral administration of the $\mathrm{Na}(+)$-glucose cotransporter inhibitor T-1095. British Journal of Pharmacology. 2001; 132: 578-586.

24. Oku A, Ueta K, Arakawa K, et al. Correction of hyperglycemia and insulin sensitivity by T-1095, an inhibitor of renal $\mathrm{Na}+$-glucose cotransporters, in streptozotocin-induced diabetic rats. Jpn J Pharmacol. 2000; 84: 351-354.

25. Ueta K, Ishihara T, Matsumoto $Y$, et al. Long-term treatment with the Na+-glucose cotransporter inhibitor T-1095 causes sustained improvement in hyperglycemia and prevents diabetic neuropathy in Goto-Kakizaki Rats. Life Sci. 2005; 76: 2655-2668.

26. Nawano M, Oku A, Ueta K, et al. Hyperglycemia contributes insulin resistance in hepatic and adipose tissue but not skeletal muscle of ZDF rats. American Journal of Physiology: Endocrinology and Metabolism. 2000; 278: E535-543.

27. Mizuma T, Hagi K, and Awazu S. Intestinal transport of beta-thioglycosides by $\mathrm{Na}+$ /glucose cotransporter. J Pharm Pharmacol. 2000; 52: 303-310.

28. Hirayama BA, Lostao MP, Panayotova-Heiermann M, et al. Kinetic and specificity differences between rat, human, and rabbit $\mathrm{Na}+$ - glucose cotransporters (SGLT-1). American Journal of Physiology: Gastrointestinal and Liver Physiology. 1996; 270: G919-G926.

29. Castaneda F, and Kinne RKH. A 96-well automated method to study inhibitors of human sodium-dependent D-glucose transport. Molelcular and Cellular Biochemistry. 2005; 280: 91-98.

30. Shirota K, Kato Y, Suzuki K, et al. Characterization of novel kidney-specific delivery system using an alkylglucoside vector. J Pharmacol Exp Ther. 2001; 299: 459-467.

31. Feld BK, Pasteels JM, and Boland W. Phaedon cochleariae and Gastrophysa viridula (Coleoptera:Chrysomelidae) produce defensive iridoid monoterpenes de novo and are able to sequester glycosidically bound terpenoid precursors. Chemoecology. 2001; 11: 191-198.

32. Kuhn J, Pettersson EM, Feld BK, et al. Selective transport systems mediate sequestration of plant glucosides in leaf beetles: A molecular basis for adaptation and evolution. Proc Natl Acad Sci U S A. 2004; 101: 13808-13813.

33. Kuhn J, Pettersson EM, Feld BK, et al. Sequestration of Plant-Derived Phenolglucosides by Larvae of the Leaf Beetle Chrysomela populi: Thioglucosides as Mechanistic Probes. In press.

34. Scheen AJ. Is there a role for alpha-glucosidase inhibitors in the prevention of type 2 diabetes mellitus? Drugs. 2003; 63: 933-951.

35. Suzuki K, Susaki H, Okuno S, et al. Renal drug targeting using a vector "alkylglycoside". J Pharmacol Exp Ther. 1999; 288: 57-64.

36. Suzuki K, Ando T, Susaki H, et al. Structural requirements for alkylglycoside-type renal targeting vector. Pharm Res. 1999; 16: 1026-1034.

37. Vestri S, Okamoto MM, de Freitas HS, et al. Changes in sodium or glucose filtration rate modulate expression of glucose transporters in renal proximal tubular cells of rat. Journal of Membrane Biology. 2001; 182: 105-112.

38. Meyer C, Stumvoll M, Nadkarni V, et al. Abnormal renal and hepatic glucose metabolism in type 2 diabetes mellitus. J Clin Invest. 1998; 102: 619-624.

39. Dominguez JH, Camp K, Maianu L, et al. Molecular adaptations of GLUT1 and GLUT2 in renal proximal tubules of diabetic rats. 
Am J Physiol. 1994; 266: F283-290.

40. Burckhardt G, and K.H KR. Transport proteins. Cotransporters and countertransportes. In: Handbook of Physiology - Renal Physiology. New York: Oxford University Press. 1992: 2083-2117.

41. Au A, Gupta A, Schembri P, et al. Rapid insertion of GLUT2 into the rat jejunal brush-border membrane promoted by glucagon-like peptide 2. Biochemical Journal. 2002; 367: 247-254.

42. Marks J, Debnam ES, Dashwood MR, et al. Detection of glucagon receptor mRNA in the rat proximal tubule: potential role for glucagon in the control of renal glucose transport. Clin Sci (Lond). 2003; 104: 253-258.

43. Diez-Sampedro A, Lostao MP, Wright EM, et al. Glycoside binding and translocation in $\mathrm{Na}(+)$-dependent glucose cotransporters: comparison of SGLT1 and SGLT3. The Journal of Membrane Biology. 2000; 176: 111-117.

44. Santer R, Kinner M, Lassen CL, et al. Molecular analysis of the SGLT2 gene in patients with renal glucosuria. Journal of the American Society of Nephrology. 2003; 14: 2873-2882. 